\title{
35 A CELLULAR NEURAL NETWORK FOR DEFORMABLE OBJECT MODELLING
}

\author{
Y. Zhong ${ }^{1}$, B. Shirinzadeh ${ }^{1}$, X. Yuan ${ }^{2}$, G. Alici ${ }^{3}$ and J. Smith ${ }^{4}$ \\ 'Department of Mechanical Engineering, Monash University, Australia \\ ${ }^{2}$ School of Computer Science, University of Windsor, Canada \\ ${ }^{3}$ School of Mechanical, Materials, and Mechatronics Engineering \\ University of Wollongong, Australia \\ ${ }^{4}$ Monash Medical Centre, Department of Surgery, Monash University, Australia
}

\begin{abstract}
This paper presents a new methodology for the deformation of soft objects by drawing an analogy between cellular neural network (CNN) and elastic deformation. An improved CNN model is developed to simulate the deformation of soft objects. A finite volume based method is presented to derive the discrete differential operators over irregular nets for obtaining the internal elastic forces. The proposed methodology not only models the deformation dynamics in contimum mechanics, but it also simplifies the complex deformation problem with simple setting CNN templates.
\end{abstract}

\section{INTRODUCTION}

Deformable object modelling is essential for many industrial and medical applications such as assembly and disassembly of flexible parts, and surgery simulation. To this end, a significant amount of research efforts have been dedicated to simulating the behaviours of deformable objects. These research efforts can be divided into two classes. The first class of studies is focused on real-time simulation such as mass-spring models [Choi et al, 2003] and spline surfaces used for deformation simulation and visualization [Bockholt et al, 1999]. The advantage of these methods is that the computation is less time consuming and the algorithm is easier to be implemented. However, the method does not allow accurate modelling of material properties, and more importantly, increasing the number of springs leads to a stiffer system. The other class of investigations focuses on deformation modelling using techniques such as Finite Element Method (FEM) [Basdogan et al, 2004] and Boundary Element Method (BEM) [Monserrat et al, 2001]. In FEM or $\mathrm{BEM}$, rigorous mathematical analysis based on continuum mechanics is applied to accurately model the mechanical behaviours of deformable objects. However, these methods are computationally expensive and are typically simulated off-line. The pre-calculation [Monserrat et al, 2001], matrix condensation [Bro-Nielsen, 1998], the space and time adaptive level-of-detail [Debunne et al, 2001] and explicit finite element [Cotin et al, 2000] techniques are used to enhance the computational performance.

Please use the following format when citing this chapter:

Zhong, Y., Shirinzadeh, B., Yuan, X., Alici, G., Smith, J., 2006, in IFIP International Federation for Information Processing, Volume 220, Information Technology for Balanced Manufacturing Systems, ed. Shen, W., (Boston: Springer), pp $329-336$ 
There are several investigations that combine neural network with deformable modelling [Nurnberger et al, 2001; Duysak et al, 2003]. However, in these methods, neural networks are mainly used to determine the parameters of mass-spring models. To the best of our knowledge, this study is the first to directly use neural network techniques to mimic the behaviours of deformable objects under externally applied loads.

This paper presents a new methodology for accurate modelling deformable objects by drawing an analogy between cellular neural network (CNN) and elastic deformation. The deformation is formulated as a dynamic CNN. An improved autonomous $\mathrm{CNN}$ model is developed to mimic the deformation of soft objects through the CNN activity. The internal elastic forces are incorporated in the CNN model as the local connectivity of cells and the external applied force as the independent current source to model the deformation dynamics. A finite volume based method is presented to derive the discrete differential operators over irregular nets. The finite volume method enforces the conservation of energy in a discrete sense and provides an intuitively geometric discretization rather than an interpolating function in the finite element method to calculate internal elastic forces. The proposed methodology not only models the physical dynamics of soft object deformation, but it also simplifies the complex deformation problem with simple setting CNN templates.

\section{DESIGN OF CNN MODEL}

\subsection{CNN Analogy}

A CNN model can be applied to different grid types. Without loss of generality, we consider a CNN on a rectangular grid with $\mathrm{M}$ rows and $\mathrm{N}$ columns. Each node on the grid is occupied by a cell. The dynamics of the array of $\mathrm{M} \times \mathrm{N}$ cells is described by the following equation and conditions [Chua and Yang, 1988]:

$$
C \frac{d v_{x i j}(t)}{d t}=-\frac{1}{R_{x}} v_{x i j}(t)+\sum_{(k, l) \in N_{r}(i, j)} A(i, j ; k, l) v_{y k l}(t)+I_{i j}
$$

where $(i, j)$ refers to the cell associated with the node under consideration, $(k, l)$ to a cell in the neighborhood of the cell $(i, j)$, namely $N_{r}(i, j)$, within a radius $r$ of the cell $(i, j)$ ( $r=1$ for simplicity). $C$ is the capacitance of a linear capacitor, $R_{x}$ is the resistance of a linear resistor, $I_{i j}$ is the current of the independent linear/nonlinear current source, and $A$ is the feedback template. $v_{u i j}(t), v_{x i j}(t)$, and $v_{y i j}(t)$ denote the input, state and output of the cell $(i, j)$ at the time $t$, respectively. $v_{y i j}(t)$ is a nonlinear function of $v_{x i j}(t)$.

One significant feature of CNN, as well as the basic difference from other neural networks, is the local connectivity of cells [Chua and Yang, 1988], i.e. any cell in $\mathrm{CNN}$ is connected only to its neighbouring cells. Adjacent cells directly interact with each other. Cells not directly connected to each other have indirect effect because of the propagation effects of the continuous-time dynamics of CNN. 
Therefore, given the initial state and the external environment, CNN activity is only determined by the local connectivity of cells. The local connectivity of cells is similar to the internal force since the deformation is only determined by the internal force under the given external force and the initial state.

Another significant feature of CNN is that the individual cells are non-linear dynamical systems, but that the coupling between them, i.e. the local connectivity of cells, is linear [Slavova, 2003]. The feature makes CNN very suitable for modelling non-linear materials since $\mathrm{CNN}$ conserves the physical properties of the continuous structure.

Further, CNN offers an incomparable computation speed due to the collective and simultaneous activity of all cells [Roska et al, 1995; Kozek et al, 1995]. The computation advantage of $\mathrm{CNN}$ is very suitable for real-time the computation requirement of deformable object simulation.

In the proposed $\mathrm{CNN}$ analogy, the deformation of soft objects is treated as the activity of a CNN. The object surface is treated as a CNN by using a number of locally connected cells. The external force is treated as the current source of the contact cell. The local interactions generated by the local connectivity of cells are treated as internal forces, and the CNN dynamic activity is treated as the dynamics of deformation. Therefore, such a CNN with the current source, the local connectivity and the dynamic activity can be seen as a communication medium among an external force, internal forces and deformation.

\subsection{CNN Model for Deformation}

The dynamics of soft object deformation is usually described by the Lagranage dynamics. Therefore, the CNN model is formulated as the Lagrange dynamics to describe the dynamics of soft object deformation.

When an external force is applied to a soft object, the contact point of the external force is replaced with a new position. As a result, the other points not influenced by the external force are in an unstable state. The external force is propagated among mass points to establish a new equilibrium state by generating the corresponding internal forces. Based on the equilibrium state, the new position of each point is obtained. The dynamic deformation process is governed by the Lagrangian equation, which is to balance the externally applied force with the internal forces due to the deformation of the soft object. The Lagrange equation that governs the motions of each node is [Goldsteln, 1980]:

$$
m_{i} \frac{d^{2} \mathbf{U}_{i}}{d t^{2}}+\gamma_{i} \frac{d \mathbf{U}_{i}}{d t}+\mathbf{G}_{i}=\mathbf{F}_{i}
$$

where $\mathbf{U}_{i}$ is the position vector of node $i$ at time $t$, and $m_{i}$ and $\gamma_{i}$ are the mass and damping constants of node $i$, respectively, $\mathrm{F}_{i}$ is the external force applied to node $i$ at time $t$, and $\mathbf{G}_{i}$ is the net internal force applied to node $i$ at time $t$. In the case of linear elasticity, the linear strain makes the internal force linear with respect to the position vector, i.e.

$$
\mathbf{G}_{i}=K_{i} \mathbf{U}_{i}
$$


where $K_{i}$ is a coefficient at node $\mathrm{i}$, which is related with the stiffness of a material.

The second-order differential equations can be divided into a system of first order differential equations by introducing a velocity function $\mathbf{V}_{i}$.

$$
m_{i} \frac{d \mathbf{V}_{i}}{d t}=\mathbf{F}_{i}-K_{i} \mathbf{U}_{i}-\gamma_{i} \mathbf{V}_{i} \quad \frac{d \mathbf{U}_{i}}{d t}=\mathbf{V}_{i}
$$

According to Eq. (4), the $\mathrm{CNN}$ model for dynamic deformations is designed as a second-order autonomous CNN:

$$
C \frac{d v_{x i j}(t)}{d t}=-\frac{1}{R_{x}} v_{x i j}(t)+\sum_{(k, l) \in N,(i, j)} A(i, j ; k, l) v_{y k i}(t)+I_{i j} \quad \frac{d v_{x i j}}{d t}=v_{x i j}
$$

where

$$
v_{y i j} \rightarrow \mathbf{U}_{i j} \quad v_{x i j} \rightarrow \mathbf{V}_{i j} \quad I_{i j} \rightarrow \mathbf{F}_{i j} \quad C=m_{i} \quad R=\frac{1}{\gamma_{i}}
$$

In most of CNN applications, the independent current source of each cell has the same value. For the purpose of soft object deformation, the independent current source is only set by the external force. Therefore, the current source value at the contact cell is directly obtained from the external force, and the current source values of other cells are set to zero since there is no external force applied to them.

Due to the fact that any $\mathrm{CNN}$ has only one output, a three-lay CNN is constructed to compute the $\mathrm{X}, \mathrm{Y}$ and $\mathrm{Z}$ coordinates of displacement vectors.

The solution of the Lagrangian equation needs initial values and boundary conditions. The initial values and boundary conditions should also be incorporated in the CNN model. The initial values can be directly associated with the initial state of the CNN. The simplest boundary condition is the Dirichlet boundary condition, i.e. the given boundary values. The Dirichlet boundary condition is realized by using fixed-state cells.

\section{FORMULATION OF LOCAL CONNECTIVITY OF CELLS}

From the above section, it is obvious that the local connectivity of cells is related to the internally elastic forces. A straightforward approach to derive the internal elastic force is the Hooke's law, and the relationship between the displacement $u$ and the applied internal elastic force F is [Timoshenko and Goodier, 1970]:

$$
\mathbf{F}=S[\mu \Delta \mathbf{u}+(\lambda+\mu) \nabla(\nabla \bullet \mathbf{u})]
$$

where $\Delta$ is the Laplace operator, $\nabla$ is the gradient operator, $\nabla \bullet$ is the divergence operator and $\mathrm{S}$ is the measure of the area the force applied on.

To get the internal elastic force at each node for setting the local connectivity of cells, Laplace operator and the gradient-of-divergence operator has to be discretized on the object surface. The discretization of these operators on a regular net is straightforward, and can be easily done by the techniques of finite difference. The difficulty is how to discretize these operators on an irregular net. 


\subsection{Finite Volume Method for Discrete Operators}

For the discretization of the operators on an irregular net, a finite volume method [Versteeg and Malalasekera, 1995] is used to aid establishing the discretization at each node. The finite volume method has the strength of dealing with unstructured grids and has been widely used in computational fluid and heat transfer. One straightforward finite volume method is Voronoi diagram [Barth, 1992], which derives the discretized equation at each node from the energy conservation law. It subdivides the domain into a finite number of non-overlapping cells or control volumes, over which the conservation of energy is enforced in a discrete sense. Fig. 1(a) shows the finite volume constructed by the Voronoi scheme. The finite volume of point $\mathbf{P}_{0}$ is constructed by connecting each intersection points between the perpendicular centerlines of each edges adjacent to point $\mathbf{P}_{0}$. Fig. 1(b) shows a segment of the Voronoi finite volume.

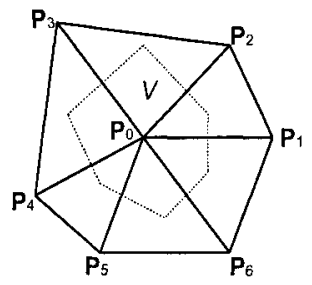

(a) A Voronoi finite volume

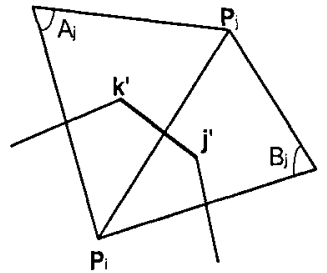

(b) A segment of the finite volume Figure 1 - Voronoi finite volume on a triangular net

By derivations, Laplace operator at $\mathbf{P}_{\mathbf{i}}$ may be written as:

$$
(\Delta \mathbf{u})_{\mathbf{P}_{i}}=\frac{1}{2 S} \sum_{j \in N_{i}}\left(\cot A_{j}+\cot B_{j}\right)\left(\mathbf{P}_{j}-\mathbf{P}_{i}\right)
$$

where $S$ is the measure of the finite volume, and $N_{i}$ is the set of the neighbour points of point $\mathbf{P}_{i}$ (see Fig. 1(b)).

By derivations, the gradient-of-divergence operator at point $\mathbf{P}_{\mathrm{i}}$ may be written as:

$$
\nabla(\nabla \bullet \mathbf{u})=\frac{1}{4 S^{2}} \sum_{j \in N_{i}}\left(\sum_{k \in N, \cap k \notin N_{i}}\left(\mathbf{u}_{j}+\mathbf{u}_{k}\right) \bullet \mathbf{n}_{\overrightarrow{\mathbf{P}_{j} \mathbf{P}_{k}}}\right) \mathbf{n}_{\overrightarrow{\mathbf{P}_{i} \mathbf{P}_{j}}}
$$

where $\mathbf{n}_{\overrightarrow{\mathbf{P}_{i} \mathbf{P}_{j}}}=\frac{\overrightarrow{\mathbf{P}_{i} \mathbf{P}_{j}}}{\left\|\overrightarrow{\mathbf{P}_{i} \mathbf{P}_{j}}\right\|}$.

\subsection{Formulation for the Local Connectivity of Cells}

The local connectivity of cells is set by the internally elastic forces. Replacing Eqs. (8) and (9) in Eq. (7), the internal force at $\mathbf{P}_{\mathbf{i}}$ is: 
$\mathbf{F}_{i}=\frac{1}{2} \sum_{j \in N_{i}}\left(\mu\left(\cot A_{j}+\cot B_{j}\right)+\frac{\lambda+\mu}{2 S\left\|\overrightarrow{\mathbf{P}_{i} \mathbf{P}_{j}}\right\|} \sum_{k \in N_{j} \cap k \in N_{i}}\left(\mathbf{P}_{j}+\mathbf{P}_{k}\right) \bullet \mathbf{n}_{\overrightarrow{\mathbf{P}_{j} \mathbf{P}_{k}}}\right)\left(\mathbf{P}_{j}-\mathbf{P}_{i}\right)$

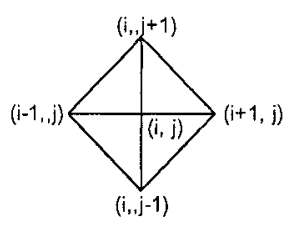

Figure $2-A$ triangular net

From Eq. (10), the local connectivity of the CNN model, i.e. the template A can be obtained. For example, assuming the object surface is a net shown in Fig. 2, the template $\mathrm{A}$ is:

$$
\begin{aligned}
& A_{i, j, i, j}=-\frac{1}{2} \sum_{m, r \in N_{i, j}}\left(\mu\left(\cot A_{m, n}+\cot B_{m, n}\right)+\frac{\lambda+\mu}{2 S\left\|\mathbf{P}_{m, n}-\mathbf{P}_{i, j}\right\|} \|_{k, l \in N_{m, n} \wedge k, l \in N_{i, j}}\left(\mathbf{P}_{m, n}+\mathbf{P}_{k, l}\right) \bullet \frac{\mathbf{P}_{k, l}-\mathbf{P}_{m, n}}{\left\|\mathbf{P}_{k, l}-\mathbf{P}_{m, n}\right\|}\right) \\
& A_{i, j, i-1, j}=\frac{1}{2}\left(\mu\left(\cot A_{i-1, j}+\cot B_{i-1, j}\right)+\frac{\lambda+\mu}{2 S\left\|\mathbf{P}_{i-1, j}-\mathbf{P}_{i, j}\right\|} \sum_{k, l \in N_{i-1, j} \sim k, l \in N_{i, j}}\left(\mathbf{P}_{i-1, j}+\mathbf{P}_{k, j}\right) \cdot \frac{\mathbf{P}_{k, j}-\mathbf{P}_{i-1, j}}{\left\|\mathbf{P}_{k, l}-\mathbf{P}_{i-1, j}\right\|}\right) \\
& A_{i, j, i+1, j}=\frac{1}{2}\left(\mu\left(\cot A_{i+1, j}+\cot B_{i+1, j}\right)+\frac{\lambda+\mu}{2 S\left\|\mathbf{P}_{i+1, j}-\mathbf{P}_{i, j}\right\|} \sum_{k, l \in N_{i+1, j} \wedge k, l \in N_{t, j}}\left(\mathbf{P}_{i+1, j}+\mathbf{P}_{k, l}\right) \bullet \frac{\mathbf{P}_{k, l}-\mathbf{P}_{i+1, j}}{\left\|\mathbf{P}_{k, l}-\mathbf{P}_{i+1, j}\right\|}\right) \\
& A_{i, j, i, j-1}=\frac{1}{2}\left(\mu\left(\cot A_{i, j-1}+\cot B_{i, j-1}\right)+\frac{\lambda+\mu}{2 S\left\|\mathbf{P}_{i, j-1}-\mathbf{P}_{i, j}\right\|} \sum_{k, l \in N_{i, j-1} \cap k, l \in N_{i, j}}\left(\mathbf{P}_{i, j-1}+\mathbf{P}_{k, l}\right) \bullet \frac{\mathbf{P}_{k, l}-\mathbf{P}_{i, j-1}}{\left\|\mathbf{P}_{k, l}-\mathbf{P}_{i, j-1}\right\|}\right) \\
& A_{i, j, i, j+1}=\frac{1}{2}\left(\mu\left(\cot A_{i, j+1}+\cot B_{i, j+1}\right)+\frac{\lambda+\mu}{2 S\left\|\mathbf{P}_{i, j+1}-\mathbf{P}_{i, j}\right\|_{k, l \in N_{t, j+l} \wedge k, l \in N_{t, j}}}\left(\mathbf{P}_{i, j+1}+\mathbf{P}_{k, l}\right) \cdot \frac{\mathbf{P}_{k, l}-\mathbf{P}_{i, j+1}}{\left\|\mathbf{P}_{k, l}-\mathbf{P}_{i, j+1}\right\|}\right) \\
& A_{i, j ; i-1, j-1}=0 \quad A_{i, j ; i \cdots 1, j+1}=0 \quad A_{i, j ; i+1, j-1}=0 \quad A_{i, j ; i+1, j+1}=0
\end{aligned}
$$

\section{IMPLEMENTATION RESULTS AND DISCUSSIONS}

A prototype system has been implemented for interactive deformable object modelling with force feedback. A PHANToM haptic device is configured with the system to carry out the deformations of deformable objects with force feedback. The PHANToM is a six degree-of-freedom haptic device from Sensable Technologies, and provides force feedback to a user. A user can use the device to touch the soft object and deform it by pushing, pulling and dragging the object surface in a natural $3 \mathrm{D}$ environment.

Fig. 3 illustrates the deformations of an elliptic sphere with 400 mass points. Fig. 3(a) shows the undeformed elliptic sphere. Fig. 3(b) and (c) show different views of the object deformed under a tensile force. Fig. 4 shows the deformation modelling of a shoes-shaped object under a compressive force.

Compared to the mass-spring method, the proposed methodology is more accurate than mass-spring due to the formulation of the local connectivity of cells based on continuum mechanics. Compared to BEM and linear FEM, the proposed 
methodology has the computational advantage due to the collective and simultaneous activity of all cells. In addition, the $\mathrm{CNN}$ model is easier to be formulated than the complex BEM and linear FEM models. Only surface mass points are involved in computation and rendering without any inside points, while the interior must be meshed and calculated in FEM.

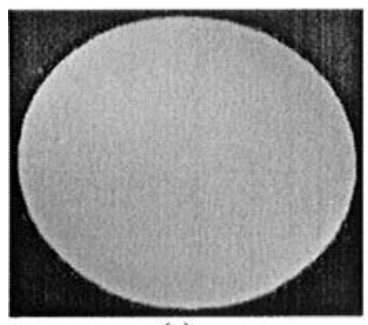

(a)

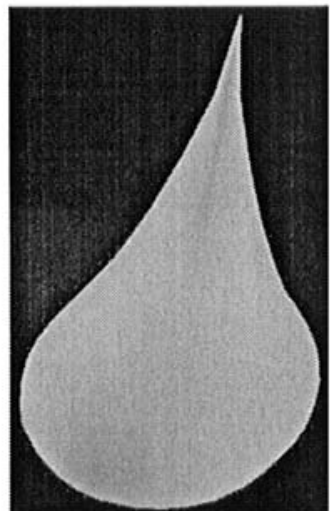

(b)

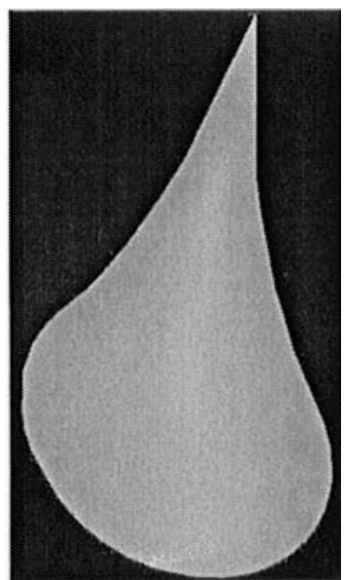

(c)

Figure 3-Deformations of an elliptic sphere

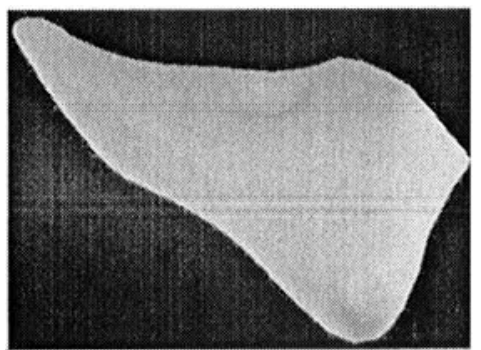

(a)

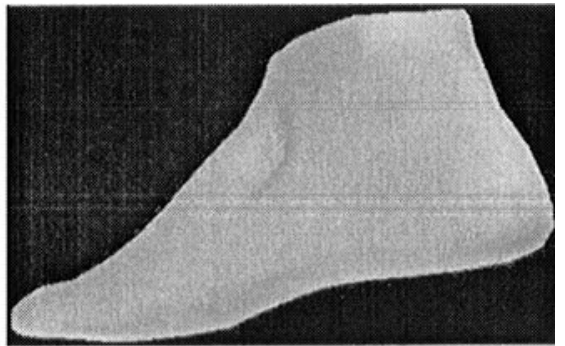

(b)

Figure 4 -Deformations of a shoes-shaped object

\section{CONCLUSIONS}

Presented in this paper is a new methodology to mimic the deformation of soft objects by drawing an analogy between $\mathrm{CNN}$ and elastic deformation. The contribution of this paper is that the CNN techniques are used to describe the deformation dynamics through incorporating the externally applied force and the internally elastic forces, and new discrete differential operators are derived to ensure the conservation of energy and provide the geometric intuitiveness. An improved $\mathrm{CNN}$ model is developed to describe the dynamics of soft object deformation. A finite volume based method is presented to derive the discrete differential operators 
over irregular nets for obtaining the internal elastic forces. The finite volume method enforces the conservation of energy in a discrete sense and also provides an intuitively geometric discretization rather than an interpolating function in finite element methods to calculate internal elastic forces. The proposed methodology not only models the physical dynamics of soft object deformation, but it also simplifies the complex deformation problem with simple setting CNN templates.

\section{ACKNOWLEDGEMENTS}

This paper is supported by Australian Research Council (ARC) and NSERC of Canada.

\section{REFERENCES}

1. Choi K., Sun H. and Heng P. A. Interactive deformation of soft tissues with haptic feedback for medical learning, IEEE Transaction on Information Technology in Biomedicine, vol. 7, no. 4, 2003, pp358-363

2. Bockholt U., Müller W. and Voss G. (etc.). Real-time simulation of tissue deformation for the nasal endoscopy simulator (NES), Computer aided surgery, vol. 4, no. 5, 1999, pp281-285

3. Basdogan C., De S. and Kim J. (etc.). Haptics in minimally invasive surgical simulation and training, IEEE Computer Graphics and Applications, vol. 24, no. 2, 2004, pp56-64

4. Monserrat C., Meier U. and Alcaniz M. (etc.). A new approach for the real-time simulation of tissue deformations in surgery simulation, Computer Methods and Programs in Biomedicine, vol. 64, 2001, pp77-85

5. Bro-Nielsen M. Finite element modeling in surgery simulation, Proceedings of The IEEE, vol. 86, no 3, 1998, pp490-503

6. Debunne G., Desbrun M., Cani M. P. and Barr A. H. Dynamic real-time deformations using space \& time adaptive sampling. Proceedings of the 28 th annual conference on Computer graphics and interactive techniques, Los Angeles, USA, 2001, pp31-36

7. Cotin S., Delingette $H$. and Ayache N. A hybrid elastic model allowing real-time cutting, deformations and force-feedback for surgery training and simulation, The Visual Computer, vol. 16, no. 8, 2000, pp437-452

8. Nurnberger A, Radetzky A. and Kruse R. Using recurrent neuro-fuzzy techniques for the identification and simulation of dynamic systems, Neurocomputing, vol. 36, 2001, pp123-147

9. Duysak A., Zhang J. J. and Ilankovan V. Efficient modelling and simulation of soft tissue deformation using mass-spring systems, International Congress Series, vol. 1256, 2003, pp337-342

10. Chua L. O. and Yang L., Cellular neural network: Theory, IEEE Transactions on Circuits and Systems, vol. 35, no. 10, 1988, pp1257-1272

11. Slavova A. Cellular Neural Networks: Dynamics and Modelling, Dordrecht, London: Kluwer Academic Publishers, 2003

12. Roska T., Chua L. O., Wolf D., Kozek T., Tetzlaff R. and Puffer F. Simulation nonlinear waves and partial differential equations via CNN-Part I: Basic Techniques, IEEE Transactions on Circuits and Systems, Vol. 42, No. 10, 1995, pp. 807-815

13. Kozek $T_{\text {, }}$ Chua L. O., Roska $T$, Wolf D., Tetzlaff R., Puffer F, and Lotz K. Simulating nonlinear waves and partial differential equations via $\mathrm{CNN}$-Part II: typical examples, IEEE Transactions on Circuits and Systems, vol. 42, no. 10, 1995, pp. 816-820

14. Goldsteln H. Classical Mechanics, Second Edition, Reading, MA: Addison-Wesley, 1980.

15. Timoshenko S. P. and Goodier J. N. Theory of Elasticity. McGraw-Hill, 1970

16. Versteeg H. K. and Malalasekera W. An introduction to computational fluid dynamics: the finite volume method, Harlow, Essex, England: Longman Scientific \& Technical, 1995

17. Barth T. J. Aspects of unstructured grids and finite-volume solvers for the Euler and Navier-Stokes equations, AGARD Report 787, 1992, pp6.1-6.61 